\title{
Nuevos registros de Castilleja (Orobanchaceae) en Veracruz, México
}

\section{New records of Castilleja (Orobanchaceae) in Veracruz, Mexico}

\author{
Antonio Francisco-Gutiérrez,3, Jaime Ernesto Rivera-Hernández² y Abel Felipe Vargas-Rueda²
}

1 Instituto de Ecología A.C., Red de Biodiversidad y Sistemática, Carretera Antigua a Coatepec 351, El Haya, 91070 Xalapa, Veracruz, México.

2 Centro de Estudios Geográficos, Biológicos y Comunitarios, S.C., calle Santa María 13, Unidad Habitacional San Román, 94542 Córdoba, Veracruz, México.

3 Autor para correspondencia: antoniofco52@gmail.com

Citar como

Francisco-Gutiérrez, A., J. E. Rivera-Hernández y A. F. Vargas-Rueda. 2016. Nuevos registros de Castilleja (Orobanchaceae) en Veracruz, México. Acta Botanica Mexicana 117: 101-107

Recibido el 29 de septiembre de 2015. Revisado el 11 de mayo de 2016.

Aceptado el 19 de agosto de 2016

\section{Resumen:}

Antecedentes y Objetivos: Castilleja nervata y C. tenuiflora var. xylorrhiza estaban reportadas para 11 y cinco estados de México, respectivamente. El objetivo de este estudio fue determinar los caracteres morfológicos de $C$. nervata para diferenciarla de $C$. arvensis, identificar a $C$. tenuiflora var. xylorrhiza dentro del complejo C. tenuiflora y separar este último de C. integrifolia var. integrifolia, así como mostrar la distribución geográfica y documentar la similitud morfológica de los taxones.

Métodos: Se revisaron ejemplares de herbario y se colectaron los taxones, además de que se tomaron medidas y se caracterizaron las estructuras morfológicas de los especímenes encontrados en el Parque Nacional Cañón del Río Blanco, mismos que fueron determinados con base en la literatura. Resultados clave: Se determinaron dos taxones del género Castilleja que no estaban reportados con anterioridad para la flora del estado de Veracruz, México. Se proporcionan dos cuadros comparativos para la identificación de los taxones correspondientes al complejo C. tenuiflora y para diferenciar entre $C$. arvensis y $C$. nervata, así como fotografías y un mapa de distribución de los nuevos registros. Conclusiones: Estos taxones forman parte de las 10 especies y ocho variedades del género Castilleja presentes en Veracruz. Además, se recomienda revisar el género en otros estados de México y continuar con la exploración botánica del Parque Nacional Cañón del Río Blanco.

Palabras clave: Castilleja, Castilleja nervata, Castilleja tenuiflora, Orobanchaceae, Scrophulariaceae.

\section{ABSTRACT:}

Background and Aims: Castilleja nervata and C. tenuiflora var. xylorrhiza were reported for 11 and five states of Mexico, respectively. The aim of this study was to determine the morphological characters of $C$. nervata in order to differentiate it from $C$. arvensis, to identify $C$. tenuiflora var. xylorrhiza as part of the $C$. tenuiflora complex and separate the latter from $C$. integrifolia var. integrifolia, as well as showing the geographic distribution and documenting the morphological similitude of these taxa.

Methods: Herbaria specimens were revised and the taxa were collected, in addition to measuring and characterizing the morphological structures of the specimens found in the National Park "Cañon del Río Blanco", which were determined based on literature.

Key results: Two taxa of the genus Castilleja unreported previously for the flora of the state of Veracruz, Mexico were identified. Two comparative tables for the identification of the taxa corresponding to the C. tenuiflora complex and for distinguishing between $C$. arvensis y $C$. nervata are presented. Photographs and a map of distribution of the new records are also provided.

Conclusions: These taxa belong to the 10 species and eight varieties of the genus Castilleja present in Veracruz. Additionally, we recommend to revise the genus in other Mexican states and to continue the botanical exploration of the National Park "Canón del Río Blanco".

Key words: Castilleja, Castilleja nervata, Castilleja tenuiflora, Orobanchaceae, Scrophulariaceae.

\section{INTRODUCCIÓN}

Durante el siglo XX, el género Castilleja Mutis ex L.f. fue clasificado dentro de la familia Scrophulariaceae (Eastwood, 1909; Nesom, 1992; Chuang y Heckard, 1993; Méndez-Larios y Villaseñor-Ríos, 2001). Posteriormente, como resultado del análisis de estudios moleculares, este género se transfirió a la familia Oro- 
banchaceae (Oxelman et al., 2005). Los primeros estudios sobre la distribución del género en México contabilizaron entre 26 y 54 especies (Hemsley, 1882; Eastwood, 1909). En décadas recientes, la cantidad de taxones ha oscilado entre 65 (Nesom, 1992), 67 (CONABIO, 2009) y 72 especies y dos subespecies (Méndez-Larios y Villaseñor-Ríos, 2001). En lo que refiere a entidades federativas, se documentaron 16 especies del género para el estado de Oaxaca (Méndez-Larios y Hernández, 1992), cuatro para el Valle Tehuacán-Cuicatlán que abarca los estados de Puebla y Oaxaca (Alvarado-Cárdenas, 2008) y nueve en el Valle de México (Rodríguez-Jiménez, 2001). En el listado florístico realizado para el estado de Veracruz por Sosa y Gómez-Pompa (1994) se reportaron 15 especies para el género, de las cuales 10 especies y ocho variedades fueron confirmadas al concluir la revisión taxonómica para Castilleja (Francisco-Gutiérrez, 2014), siendo C. nervata Eastw. y C. tenuiflora Benth. var. xylorrhiza (Eastw.) G.L. Nesom taxones identificados como no registrados anteriormente para la entidad veracruzana. El objetivo de este estudio fue determinar las diferencias morfológicas entre algunos de los taxones presentes y los taxones no registrados de Castilleja para el estado de Veracruz.

\section{MATERIALES Y MÉTODOS}

Se siguió el procedimiento establecido por el proyecto "Flora de Veracruz" para las revisiones taxonómicas de las familias presentes en ese estado (Gómez-Pompa y Moreno, 1985), por lo que se llevó a cabo una revisión bibliográfica de las especies de Castilleja reportadas para el estado de Veracruz y se consultaron 134 especímenes herborizados resguardados en las colecciones CORU, ENCB, MEXU, XAL y XALU, de los cuales 11 pertenecieron a los taxones que aquí se presentan. Así mismo, se realizaron exploraciones botánicas para la colecta de los especímenes y su incorporación a colecciones botánicas (Lot y ChiangCabrera, 1986). Para la caracterización de los taxones se realizaron mediciones de las estructuras vegetativas y reproductivas y se determinaron con la literatura disponible. Las clasificaciones empleadas en este estudio fueron las propuestas por el Angiosperm Phylogeny Group (APG IV,
2016), a nivel específico con base en estudios moleculares propuesta por Tank y colaboradores (2009) y a nivel infraespecífico para el complejo C. tenuiflora Benth. establecida por Nesom (1992). Adicionalmente, se consultó la guía fotográfica para los taxones infraespecíficos de Castilleja tenuiflora presentada por Egger (2013).

\section{Resultados}

Durante las expediciones realizadas en el Parque Nacional Cañón del Río Blanco, en Veracruz, se localizaron y colectaron especímenes de Castilleja nervata y Castilleja tenuiflora var. xylorrhiza.

\section{Orobanchaceae}

Castilleja nervata Eastw. Proc. Amer. Acad. Arts 44(21): 574-575. 1909. TIPO: MÉXICO. Chihuahua, cercanía de Madera, alt. 2250 m. E. Palmer 274 (holotipo: GH!, isotipos: F!, S!, US!) $\equiv$ C. angustifolia M. Martens \& Galeotti, Bull. Acad. Roy. Sci. Bruxelles 12(2): 29. 1845. TIPO: MÉXICO. Michoacán, en los campos y bosques de Mirador, a 3000 pies, y al sur en la lava del malpaís del Volcán de Jorullo. Galeotti 1033, 1078, 1098 (sintipos: BR!). Not C. angustifolia (Nutt.) G. Don, Gen. Hist. 4: 616. 1838. Not C. angustifolia Gand., Fl. Eur. 18: 25. $1889 \equiv$ C. cruenta Standl., Muhlenbergia 5(6): 82-83. 1909. TIPO: ESTADOS UNIDOS DE AMÉRICA. Arizona, Chiricahua Mountains. Blumer 2133 (holotipo: NY!, isotipos: ARIZ!, US!).

Especie registrada previamente para los estados de Chihuahua, Coahuila, Durango, Jalisco, México, Michoacán, Morelos, Nayarit, Oaxaca y Sinaloa (Méndez-Larios y Villaseñor-Ríos, 2001; CONABIO, 2009; Egger, 2013). Ahora se registra del municipio de Maltrata, Veracruz (Fig. 1), en vegetación secundaria derivada de matorral xerófilo y del municipio de Huayacocotla, en matorral xerófilo.

Ejemplares examinados: MÉXICO. Veracruz, municipio de Maltrata, $600 \mathrm{~m}$ de la desviación de la autopis- 


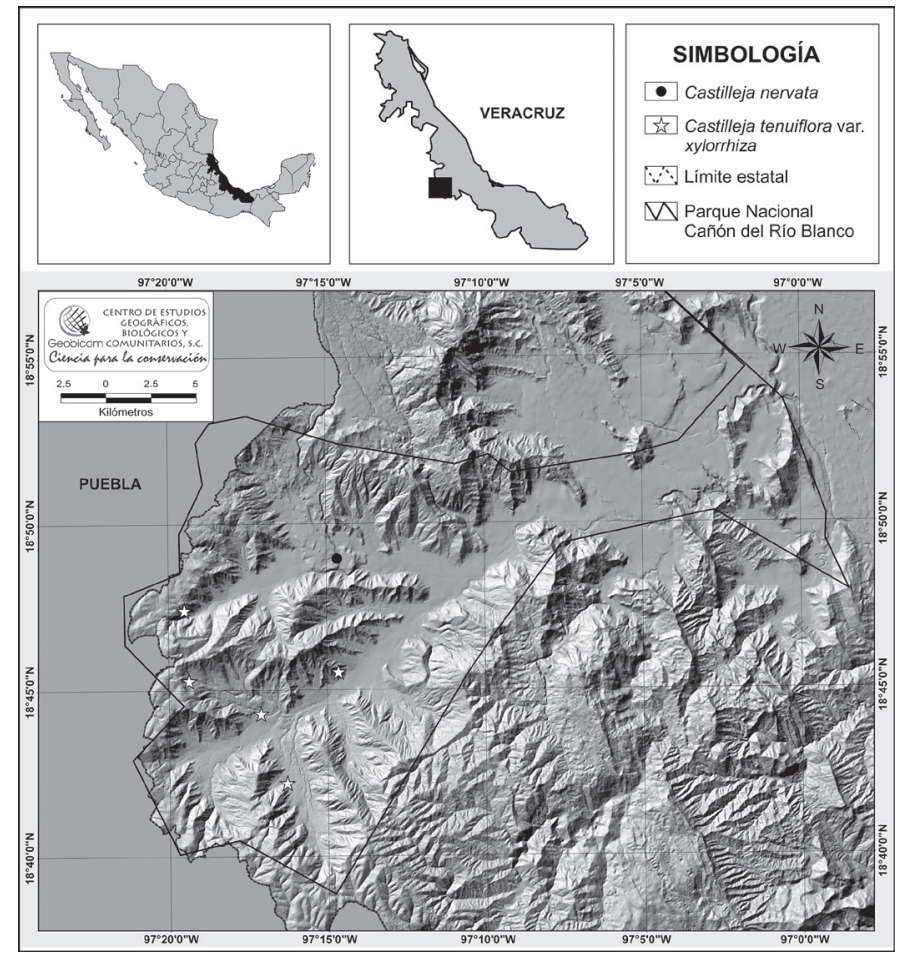

Figura 1: Mapa de distribución de $C$. nervata Eastw. y C. tenuiflora Benth. var. xylorrhiza (Eastw.) G.L. Nesom dentro del polígono correspondiente al Parque Nacional Cañón del Río Blanco en el estado de Veracruz, México. Mapa: J. E. Rivera-Hernández, modificado de INEGI (2016).

Cuadro 1: Comparación de los caracteres de C. arvensis Schltdl. \& Cham. y C. nervata Eastw.

\begin{tabular}{lcc}
\hline Caracter & C. arvensis & C. nervata \\
\hline Duración & Anual & Perenne \\
Largo de hojas & $1.9-7.5 \mathrm{~cm}$ & $2.3-4.7 \mathrm{~cm}$ \\
Ancho de hojas & $0.3-2.5 \mathrm{~cm}$ & $0.2-0.7 \mathrm{~cm}$ \\
Largo de brácteas & $0.5-2.1 \mathrm{~cm}$ & $1.2-2.2 \mathrm{~cm}$ \\
$\quad$ superiores & Amarilla, naranja, & Roja o naranja \\
Coloración brácteas & rosa, roja o púrpura & \\
$\quad$ superiores & $0.7-1.4 \mathrm{~cm}$ & $1.3-2.1 \mathrm{~cm}$ \\
Largo del cáliz & $0.3-0.5 \mathrm{~cm}$ & $0.5-1 \mathrm{~cm}$ \\
Ancho del cáliz & Cápsula circular & Cápsula ovoide \\
Forma del fruto & $0.4-0.7 \mathrm{~cm}$ & $0.8-0.9 \mathrm{~cm}$ \\
\hline Largo del fruto &
\end{tabular}

ta México - Veracruz al poblado Maltrata, $18^{\circ} 48^{\prime} 57^{\prime \prime N}$, 97²'40"W, alt. $1709 \mathrm{~m}, 7 . \mathrm{VI} .2014$, A. F. Vargas R. et al. 1212 (CORU); J. A. Francisco G. et al. 63 (MEXU, WTU, XAL, XALU).

C. nervata puede ser confundida en el campo con C. arvensis (Fig. 2), muy similar debido a la presencia de hojas linear-lanceoladas, corolas inconspicuas y brácteas superiores teñidas de color rojo (Cuadro 1).

Por otra parte, durante la revisión de los herbarios se localizó un ejemplar del municipio de Maltrata determinado por M. Egger como una variedad inédita de $C$. nervata, la cual está próxima a publicarse por él mismo (M. Egger, com. pers.).

Castilleja tenuiflora Benth. var. xylorrhiza (Eastw.) G.L. Nesom. Phytologia 73(5): 393. 1992. TIPO: MÉXICO. Coahuila, Sierra Encaruaciore, E. W. Nelson 3895 (GH!, isotipo: US!). Basiónimo: C. xylorrhiza Eastw., Proc. Amer. Acad. Arts 44: 586. 1909.

Variedad reportada como endémica de México, presente en los estados de Coahuila, Durango, Nuevo León, San Luis Potosí y Zacatecas (Egger, 2013; Nesom, 1992; Méndez-Larios y Villaseñor-Ríos, 2001). En Veracruz fue localizada en el municipio de Acultzingo (Fig. 1), en matorral xerófilo, concordando con los tipos de vegetación donde fue reportada anteriormente (Nesom, 1992), a una altitud entre los 1551 y 2370 m en una población con escasos individuos notablemente distanciados unos de otros.

Ejemplares examinados: MÉXICO. Veracruz, municipio de Acultzingo, cerro por el camino al poblado El Potrero, aprox. $3 \mathrm{~km}$ LR al SE de la parada El Mezquite, $18^{\circ} 42^{\prime} 10^{\prime \prime N}, 97^{\circ} 16^{\prime} 17^{\prime \prime W}$, alt. 1890 m, 1.IX.2012, J. E. Rivera H. y A. F. Vargas R. 4824 (CORU, XAL); Cerro Xochío, paraje La Organera, a $1 \mathrm{~km}$ al NO del poblado Sierra de Agua, 18 $45^{\prime} 34^{\prime \prime N}, 97^{\circ} 14^{\prime} 54^{\prime \prime} \mathrm{W}$, alt. $1720 \mathrm{~m}$, 14.VI.2014, A. F. Vargas R. et al. 1267 (CORU, XAL); 25.V.2013, A. F. Vargas R. et al. 547 (CORU); Cerro Xochío, Sierra de Agua, 1845'31"N, 97¹4'39"W, alt. 1551 

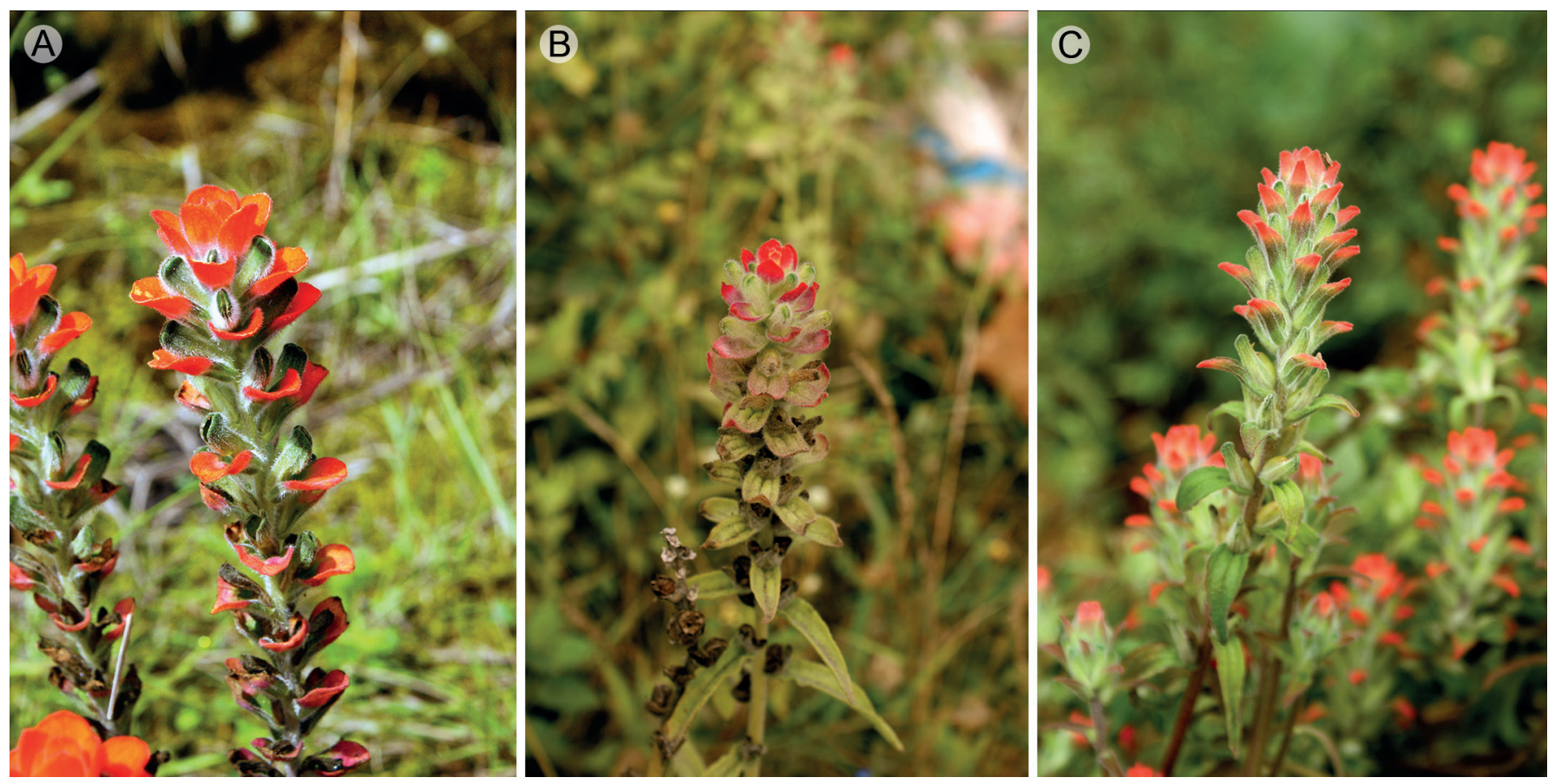

Figura 2: A. Castilleja nervata Eastw. presente en Maltrata, Veracruz. B y C. C. arvensis Schltdl. \& Cham. presente en Jáltipan y Xalapa, Veracruz, respectivamente. Fotografías: A. Francisco-Gutiérrez.

m, 28.VII.2014, J. A. Francisco G. et al. 72 (MEXU, WTU, XAL, XALU); Lindavista, $700 \mathrm{~m}$ sobre el camino de enfrente de la parada El Mezquite, $18^{\circ} 44^{\prime} 16^{\prime \prime} \mathrm{N}$, $97^{\circ} 17^{\prime} 06^{\prime \prime} \mathrm{W}$, alt. 1630 m, 20.X.2012, J. E. Rivera H. et al. 5075 (CORU, XAL); Ojo de Agua de Arriba, $5 \mathrm{~km}$ al NO LR de la parada El Mezquite, $18^{\circ} 45^{\prime} 16^{\prime \prime} \mathrm{N}, 97^{\circ} 19^{\prime} 22^{\prime \prime} \mathrm{W}$, alt. 2370 m, 20.X.2012, A. F. Vargas R. et al. 82 (CORU, XAL). Municipio de Aquila, Cumbres de Aquila, camino a las antenas de microondas, a $1.8 \mathrm{~km} \mathrm{LR}$ al O de la lo-

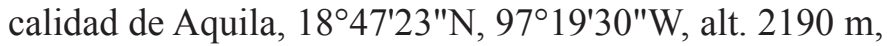
25.IV.2013, A. F. Vargas R. et al. 462 (CORU, XAL).

Es posible encontrar individuos atípicos de la variedad tenuiflora (conocida como forma "amarilla") que presentan los ápices de las brácteas, cálices y corolas amarillas (Fig. 3), primeramente documentados en el estado de Oaxaca (Egger, 2013), los cuales ahora se registran en la zona de Acultzingo, debido probablemente al flujo genético entre taxones, lo que origina la amplia variación morfológica observable en el género, de acuerdo con la hipótesis propuesta por Chuang y Heckard (1993). Estos individuos son morfológicamente muy similares a los otros miembros del complejo C. tenuiflora (C. tenuiflora var. xylorrhiza, C. tenuiflora var. tenuiflora y C. tenuiflora var. tancitaroana (G.L. Nesom) J.M. Egger) propuesto por Nesom (1992), que a su vez presentan similitud con C. integrifolia L.f. var. integrifolia (Fig. 4, Cuadro 2), única variedad presente en Veracruz de las tres variedades de esta especie.

Ejemplares adicionales examinados de Castilleja tenuiflora var. tenuiflora forma "amarilla": MÉXICO. Veracruz, municipio de Acultzingo, camino a Puente Colorado, alt. 2350 m, 06.XI.1985, J. L. Martínez y R. Acosta P. 1018 (MEXU, XAL); $4 \mathrm{~km}$ antes de Acultzingo rumbo a Orizaba, alt. 2000 m, 28.VII.1971, I. Nevling y A. Gómez P. 2182 (MEXU).

\section{DISCUSIÓN Y CONCLUSIONES}

El tratamiento taxonómico que incluye a $C$. tenuiflora var. xylorrhiza dentro del complejo C. tenuiflora fue 

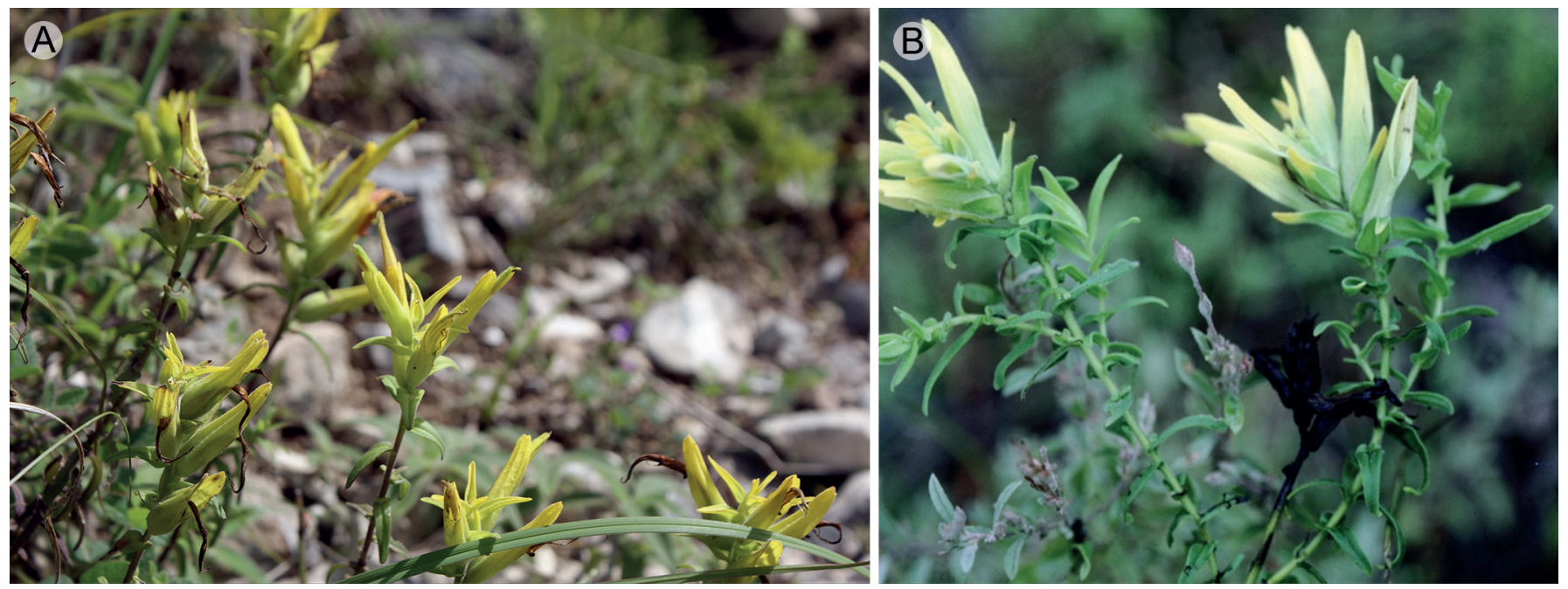

Figura 3: A. Castilleja tenuiflora Benth. var. xylorrhiza (Eastw.) G.L. Nesom presente en Acultzingo, Veracruz, B. Castilleja tenuiflora Benth. var. tenuiflora forma "amarilla" fotografiada cerca de la Ciudad de Oaxaca, Oaxaca, similar a la que se reporta en el estado de Veracruz en la zona de Cumbres de Maltrata. Fotografía A: A. Francisco-Gutiérrez y B: M. Egger.
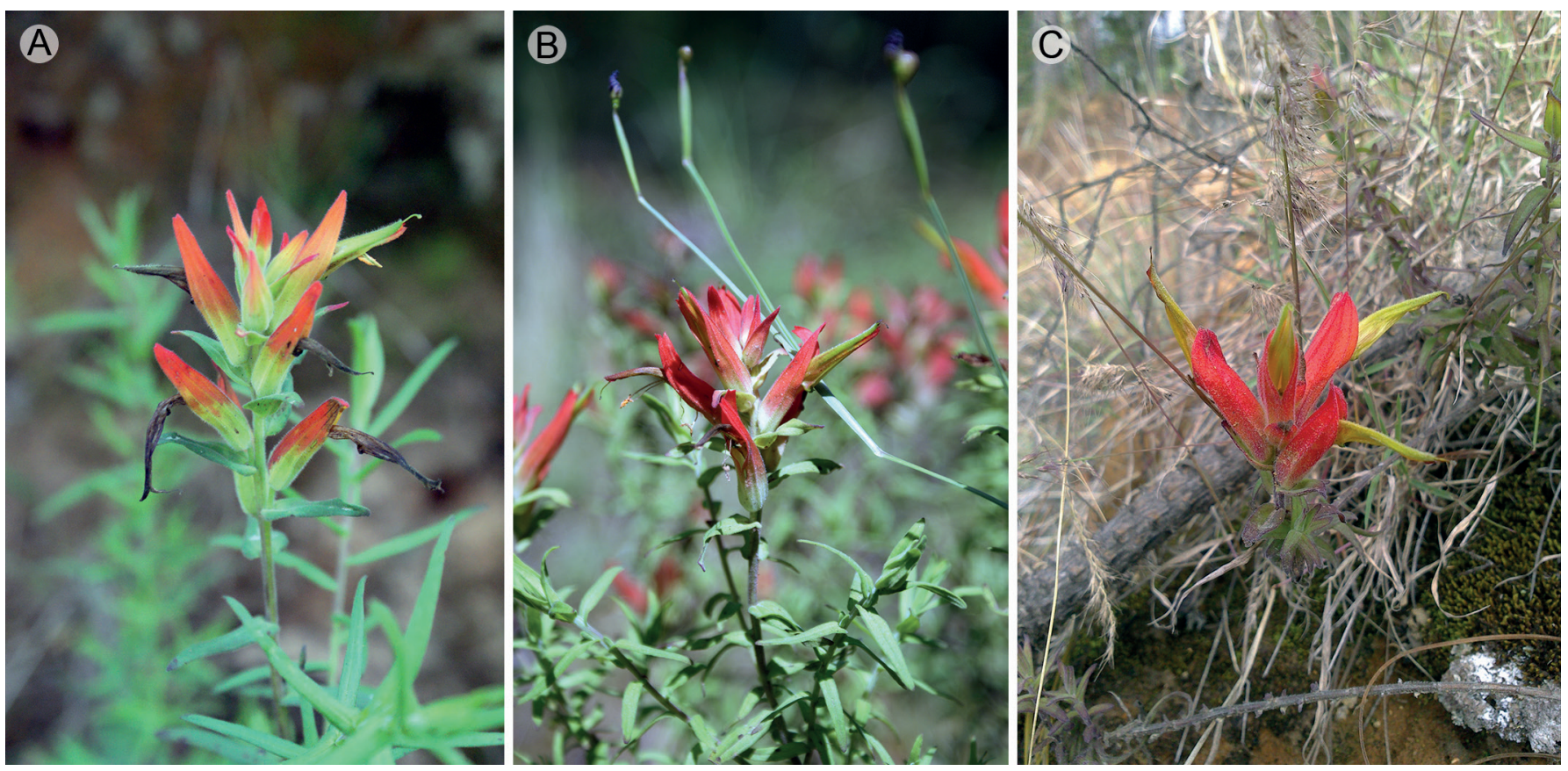

Figura 4: A. Castilleja integrifolia L.f. var. integrifolia, B. Castilleja tenuiflora Benth.var. tenuiflora en su forma típica, C. Castilleja tenuiflora var. tancitaroana (G.L. Nesom) J.M. Egger. Todas presentes en Perote, Veracruz. Fotografías: A. Francisco-Gutiérrez.

elaborado por Nesom (1992) en el cual se detectaron las siguientes situaciones. Primero, este autor reporta en la descripción de la variedad xylorrhiza reporta ejemplares amarillos como variantes dentro de la variedad tenuiflora en Veracruz, mismas que fueron identificadas en este estudio y fueron diferentes a los ejemplares de C. tenuiflora var. xylorrhiza. Los ejemplares mencionados por Nesom (1992) para Veracruz son formas atípi- 
Cuadro 2: Comparación de los caracteres de los taxones correspondientes al complejo C. tenuiflora.

\begin{tabular}{|c|c|c|c|c|c|}
\hline Caracter & $\begin{array}{c}\text { C. integrifolia } \text { var. } \\
\text { integrifolia }\end{array}$ & $\begin{array}{c}\text { C. tenuiflora } \text { var. } \\
\text { tancitaroana }\end{array}$ & $\begin{array}{c}\text { C. tenuiflora var. } \\
\text { tenuiflora forma } \\
\text { típica }\end{array}$ & $\begin{array}{c}\text { C. tenuiflora var. } \\
\text { tenuiflora forma } \\
\text { amarilla } \\
\end{array}$ & $\begin{array}{c}\text { C. tenuiflora var. } \\
\text { xylorrhiza }\end{array}$ \\
\hline Largo de hojas & $1.5-3.4 \mathrm{~cm}$ & $1.5-2 \mathrm{~cm}$ & $0.9-3.9 \mathrm{~cm}$ & $0.9-2.2 \mathrm{~cm}$ & $2.1-3.5 \mathrm{~cm}$ \\
\hline Ancho de hojas & $0.2-0.5 \mathrm{~cm}$ & $0.3-0.5 \mathrm{~cm}$ & $0.3-1 \mathrm{~cm}$ & $0.2-0.4 \mathrm{~cm}$ & $0.3-0.6 \mathrm{~cm}$ \\
\hline Largo de pedicelos & $0.1-1 \mathrm{~cm}$ & $0.1-0.3 \mathrm{~cm}$ & $0.1-0.3 \mathrm{~cm}$ & $0.3-0.4 \mathrm{~cm}$ & $0.2-0.5 \mathrm{~cm}$ \\
\hline Largo del cáliz & $1.6-3.1 \mathrm{~cm}$ & $2.4-3 \mathrm{~cm}$ & $1.3-2.8 \mathrm{~cm}$ & $2.4-3 \mathrm{~cm}$ & $1.7-2.8 \mathrm{~cm}$ \\
\hline $\begin{array}{l}\text { Tipo de tricomas del } \\
\text { cáliz }\end{array}$ & Glandulares & Glandulares & Articulados & Articulados & $\begin{array}{c}\text { Glandulares con } \\
\text { articulados }\end{array}$ \\
\hline Coloración del cáliz & Naranja & Naranja & Roja & Amarilla & Amarilla \\
\hline
\end{tabular}

cas amarillas de la variedad tenuiflora, que existen en áreas cercanas a las zonas donde ocurren las plantas con coloración típica roja o naranja y que coexisten también con C. tenuiflora var. xylorrhiza, de acuerdo con lo reportado por Egger (2013). Segundo, al final del trabajo, en la clave dicotómica menciona la distribución de la variedad xylorrhiza en los estados de Durango, Coahuila, Nuevo León, San Luis Potosí y Zacatecas mientras que para la variedad tenuiflora reporta su presencia desde Sonora a Nuevo León hasta Jalisco, Veracruz y Oaxaca. Méndez-Larios y Villaseñor-Ríos (2001) reportan a esta última variedad como endémica del estado de Nuevo León; de esta manera, con la localización y comparación de los taxones, se determinó a C. tenuiflora var. xylorrhiza como nuevo registro para la flora de Veracruz. Para el caso de C. nervata Méndez-Larios y Villaseñor-Ríos (2001), señalan su distribución para los estados de Chihuahua, Coahuila, Durango, Jalisco y Nayarit; sin embargo, esta especie fue localizada en Veracruz, donde se registró un solo individuo en la zona del valle de Maltrata, a orilla de la carretera, en zonas cercanas de matorral xerófilo.

Se sugiere continuar con las revisiones del género en otras zonas del país para aplicar las correcciones taxo- nómicas necesarias a nivel infraespecífico para este grupo que es morfológicamente confuso y complejo. Por otra parte, es necesario continuar con la exploración botánica en el Parque Nacional Cañón del Río Blanco, en especial en la zona de matorrales xerófilos de los municipios de Maltrata y Acultzingo, los cuales habían permanecido inexplorados hasta hace poco tiempo y donde recientemente se han reportado 26 nuevos registros para el estado de Veracruz, pertenecientes a diferentes familias (RiveraHernández et al., 2014; Rivera-Hernández et al., 2015; Torres-Cantú et al., en prensa).

\section{AgradeCIMIENTOS}

Los autores agradecen a Miguel Cházaro Basáñez y Feliza Ramón Farías por la revisión, vinculación y financiamiento que hicieron posible este trabajo y a Carlos Arzaba Villalba por su compañía en las expediciones en el Parque Nacional Cañón del Río Blanco. Gracias también a los encargados de los herbarios CORU, MEXU y XAL por las facilidades otorgadas para el préstamo y análisis de ejemplares presentes en sus instalaciones. Se agradece a Mark Egger por facilitar la imagen de la forma atípica de C. tenuiflora var. tenuiflora que muy pocas veces se ha documentado en campo. 


\section{LITERATURA CITADA}

Alvarado-Cárdenas, L. O. 2008. Orobanchaceae. Flora del Valle Tehuacán-Cuicatlán 65: 1-51.

APG IV. 2016. An update of the Angiosperm Phylogeny Group classification for the orders and families of flowering plants: APG IV. Botanical Journal of the Linnean Society 181: 1-20.

CONABIO. 2009. Catálogo taxonómico de especies de México. In: Ocegueda, S. y J. Llorente-Bousquets (coords.). Capital Natural de México. Vol. I. CD 1. Comisión Nacional para el Conocimiento y Uso de la Biodiversidad. México, D.F., México.

Chuang, T. I. y L. R. Heckard. 1993. Chromosome numbers of neotropical Castilleja (Scrophulariaceae: tribe Pedicularideae) and their taxonomic implications. Annals of the Missouri Botanical Garden 80: 974-986.

Eastwood, A. 1909. Synopsis of the Mexican and Central American species of Castilleja. Contributions from The Gray Herbarium of Harvard University 36: 563-591.

Egger, J. M. 2013. The genus Castilleja. Photo sets for a large number of species and varieties, with text commentary about each taxon. http://www.flickr.com/photos/mark egger_castilleja/collections/72157617709816218/ (consultado octubre de 2013).

Francisco-Gutiérrez, J. A. 2014. El género Castilleja Mutis ex L.f. (Orobanchaceae) en el estado de Veracruz, México. Tesis de licenciatura. Facultad de Biología, Universidad Veracruzana. Xalapa, México. 119 pp.

Gómez-Pompa, A. y N. P. Moreno (eds.). 1985. Guía para los colaboradores de la Flora de Veracruz. Instituto Nacional de Investigaciones sobre Recursos Bióticos y Field Museum of Natural History. Xalapa, México. 23 pp.

Hemsley, W. B. 1882. Labiatae. Biologia Centrali-Americani. Botany 2: 459-463.

INEGI. 2016. Datos de relieve. Continuo de Elevaciones Mexicano 3.0 (CEM 3.0). versión 2016.03.03 1340. Instituto Nacional de Geografía e Informática. México. http:// www.inegi.org.mx/geo/contenidos/datosrelieve/continental/descarga.aspx (consultado junio de 2016).

Lot, A. y F. Chiang-Cabrera (comps.). 1986. Manual de herbario, administración y manejo de colecciones, técnicas de recolección y preparación de ejemplares botánicos. Con- sejo Nacional de la Flora de México, A.C. México, D.F., México. 142 pp.

Méndez-Larios, I. y H. M. Hernández. 1992. Géneros de Scrophulariaceae en Oaxaca, México. Anales del Instituto de Biología de la Universidad Nacional Autónoma de México, serie Botánica 63(1): 31-65.

Méndez-Larios, I. y J. L. Villaseñor-Ríos. 2001. La familia Scrophulariaceae en México: diversidad y distribución. Boletín de la Sociedad Botánica de México. 69: 101-121.

Nesom, G. L. 1992. Taxonomy of the Castilleja tenuiflora group (Scrophulariaceae) in Mexico, with an overview of sect. Castilleja. Phytologia 73(5): 389-415.

Oxelman, B., P. Kornhall, R. G. Olmstead y B. Bremer. 2005. Disintegration of Scrophulariaceae. Taxon 54(2): 411 425 .

Rivera-Hernández, J. E., M. Cházaro-Basáñez, A. F. VargasRueda, F. Ramón-Farías, H. Oliva R. y G. Alcántara S. 2015. Nuevas adiciones para la flora de Veracruz. Acta Botanica Mexicana 112: 45-65.

Rivera-Hernández, J. E., J. Reyes-Santiago, M. Cházaro-Basáñez, F. Ramón-Farías, A. F. Vargas-Rueda y G. Alcántara. 2014. Las cactáceas del municipio de Acultzingo, Veracruz. Cactáceas y Suculentas Mexicanas 59(3): 68-78.

Rodríguez-Jiménez, C. 2001. Scrophulariaceae. In: Rzedowski, J. y G. C. de Rzedowski (eds.). Flora Fanerogámica del Valle de México. Comisión Nacional para el Uso y Conocimiento de la Biodiversidad e Instituto de Ecología, A.C. Pátzcuaro, México. 674-699.

Sosa, V. y A. Gómez-Pompa (eds.). 1994. Lista Florística. Flora de Veracruz 82: 1-245

Tank, D., J. M. Egger y R. G. Olmstead. 2009. Phylogenetic classification of subtribe Castillejinae (Orobanchaceae). Systematic Botany 34(1): 182-197.

Torres-Cantú, G., J. E. Rivera-Hernández, A. Badía, M. Cházaro-Basañez y A. F. Vargas-Rueda. En prensa. Nuevas adiciones para la familia Bromeliaceae en Veracruz. Botanical Sciences. 